\title{
¿Y si nos consultan porque moja la cama?
}

\author{
MI. Úbeda Sansano ${ }^{a}$, R. Martínez García ${ }^{b}$ \\ aPediatra. CS de La Eliana. Valencia. España. \\ bUrólogo. Unidad de Incontinencia Urinaria y Urología Funcional. Servicio de Urología. \\ Hospital Clínico Universitario de Valencia. España.
}

\section{Resumen}

La enuresis, equivalente al término usado con anterioridad "enuresis nocturna", es la micción involuntaria que tiene lugar durante el sueño a una edad y frecuencia socialmente inaceptables. Se acepta para el diagnóstico la edad $\geq 5$ años. Es un trastorno frecuente e infradiagnosticado, que causa una importante repercusión económica, social y emocional para muchos niños que siguen mojando la cama y sus familias. El tipo de enuresis más frecuente y de mejor pronóstico es la enuresis primaria monosintomática (EPM). Su abordaje desde Atención Primaria, con mínimas acciones diagnóstico-terapéuticas, logrará un gran impacto.

Se expondrán en el taller los aspectos clave del diagnóstico y las medidas terapéuticas disponibles para la EPM. A través de casos clínicos interactivos, el alumno será capaz de detectar precozmente este tipo de enuresis, descartar otros tipos de incontinencia y patologías asociadas y elegir el tratamiento adecuado en función de una serie de factores de buen o mal pronóstico de respuesta. Asimismo, se identificarán los casos de mala evolución que deben derivarse al hospital.

La alarma y la desmopresina son los tratamientos disponibles. Se conocerán las ventajas e inconvenientes de cada uno de ellos y la información que se debe transmitir al paciente y su familia para facilitar la adherencia al tratamiento con el mínimo riesgo.

En general, la alarma es el tratamiento más eficaz, pero su uso precisa de habilidades que se adquirirán de forma práctica en este taller.

Palabras clave: Enuresis nocturna. Niños. Incontinencia urinaria. Diagnóstico. Alarma. Desmopresina.

What if someone consult us because they wet the bed?

Abstract

Enuresis is equivalent to the former term "nocturnal enuresis". It is any involuntary discharge of urine during sleep, at a socially inappropriate age and frequency. The age of $\geq 5$ is accepted for the diagnosis. It is a frequent and under diagnosed condition that causes a relevant economic, social and emotional repercussion for a great number of relatives and chil-

M. ${ }^{\text {a }}$ Isabel Úbeda Sansano: ubeda_isa@gva.es

Los autores declaran no presentar conflictos de intereses en relación con la preparación y publicación de este artículo. 
dren that are wetting their beds. MPE (monosymptomatic primary enuresis) is the most frequent type of enuresis and has the best prognosis. The Primary Care approach will achieve a great impact with the minimal diagnosis-treatment effort.

The therapeutical measures and the key diagnostic aspects of the MPE will be exposed (explained) in the workshop. Through interactive clinical cases, the attendees will be able to detect early this type of enuresis, to rule out other type of urinary incontinence and concomitant conditions and to choose the most adequate treatment based on good or bad prognosis response factors. Likewise bad outcome cases which need to be referred to hospital will be identified.

Alarm and desmopressin are the available treatments. The attendees will learn the advantages and disadvantages of each treatment and the information that needs to be transmitted to the patient and his family to run the minimal risks and to improve the treatment adherence.

On the whole, the alarm is the more effective treatment, but an appropriated use demands specific abilities that will be acquired in a practical way in this workshop.

Key words: Child. Nocturnal enuresis. Urinary incontinence. Diagnosis. Alarm. Desmopressin.

\section{Introducción}

La enuresis es un trastorno frecuente e infradiagnosticado. Aunque su prevalencia decrece con la edad por la resolución espontánea, esta no se da en todos los casos ni en el momento deseado. Para muchas familias y niños que siguen mojando la cama, el impacto económico, social y emocional es importante.

La prevalencia de enuresis en España $a^{1,2}$ no se diferencia de la de otros países. Se estima entre el $16 \%$ a los cinco años y un 1-3\% en la adolescencia y la edad adulta, con mayor afectación del sexo masculino (1,5-2:1). El tipo más frecuente es la EPM (enuresis primaria monosintomática), que supone alrededor del $85 \%$ de los casos.
La percepción desde Atención Primaria $(A P)$ es de una prevalencia baja porque la mayoría de estos niños no están diagnosticados, bien por la tolerancia de los padres, que en muchos casos también sufrieron el problema y no consultan, bien porque no le dan importancia, o lo ocultan por vergüenza $y$, muchas veces, por la tolerancia del propio pediatra que no valora lo suficiente el problema o no tiene confianza en cómo abordarlo.

La existencia de terapias para la enuresis con eficacia probada y las características de accesibilidad y conocimiento de la familia ubican al pediatra de AP en una situación privilegiada para detectar precozmente este trastorno, tratarlo en el momento adecuado e identificar los 
casos de mala evolución para derivar al hospital. La enuresis primaria monosintomática (EPM) debe diagnosticarse y tratarse en $\mathrm{AP}$, pero para ello es necesario conocer perfectamente el tema, su magnitud y la forma de abordarlo de manera eficiente.

En este taller se siguen básicamente las recomendaciones de la "Guía de práctica clínica de enuresis nocturna primaria monosintomática en Atención Primaria" ${ }^{3}$, incluida en el catálogo de Guías de Práctica Clínica del Sistema Nacional de Salud (www.guiasalud.es /GPC/GPC_367.pdf) y en National Guideline Clearinghouse de EEUU (www. guideline.gov/content. aspx?id=12239).

\section{Objetivos}

Principal: ampliar los conocimientos para diagnosticar y tratar correctamente la enuresis. Secundarios: capacitar a los alumnos en habilidades para diagnosticar los distintos tipos de enuresis; conocer y familiarizarse con los instrumentos diagnósticos y los distintos tratamientos; identificar los casos tratables por el pediatra de AP y los que se deban derivar al hospital.

\section{Terminología}

La enuresis (enuresis nocturna) es la micción involuntaria durante el sueño a una edad y frecuencia socialmente inaceptables. En general, se acepta para el diagnóstico la edad $\geq 5$ años. Una frecuencia $<1$ noche/mes puede aceptarse en un niño pequeño, pero no en los mayores y adolescentes.

Es importante distinguir las diferentes formas de enuresis, ya que el enfoque diagnóstico y terapéutico será distinto. En la tabla 1 se describen las definiciones más útiles para nuestra práctica clínica según la terminología actual ${ }^{4,5}$ y se comentan los conceptos que pueden inducir a confusiones.

\section{Aspectos claves del diagnóstico}

\section{La anamnesis}

Es fundamental en el diagnóstico. Se recomienda realizar una entrevista dirigida a descartar otros tipos de incontinencia, a clasificar adecuadamente la enuresis (primaria o secundaria y monosintomática o no monosintomática), a valorar las patologías asociadas que puedan interferir en la evolución de la enuresis (apnea del sueño y estreñimiento) y los factores pronósticos de respuesta al tratamiento (tabla 2 ).

Puede ser de interés conocer si existen antecedentes familiares de enuresis y la existencia de tratamientos o actitudes previas y su respuesta. 


\section{La exploración física}

Es normal en la EPM. Sin embargo, para confirmar el diagnóstico y descartar problemas asociados, se recomienda una mínima exploración de:
- Abdomen (palpación de globo vesical o masas fecales por estreñimiento).

- Genitales (malformaciones, estenosis meatal en niños, adherencias la-

Tabla 1. Definiciones útiles en la enuresis. Terminología actual ${ }^{4,5}$

Enuresis (enuresis nocturna): micción involuntaria que tiene lugar durante el sueño a una edad y frecuencia socialmente inaceptables. Se acepta la edad $\geq 5$ años como límite, ya que en nuestra sociedad se espera que a esta edad ya se haya alcanzado completamente el control vesical nocturno. Generalmente, una frecuencia $<1$ noche/mes puede aceptarse en un niño pequeño, pero es inaceptable para los niños mayores y adolescentes.

Comentario: enuresis solo se debe usar como sinónimo de enuresis nocturna. No existe la enuresis diurna. Es sinónimo de incontinencia nocturna intermitente y se refiere únicamente a los episodios de incontinencia durante el sueño. El uso de enuresis en la literatura como sinónimo de incontinencia urinaria (diurna y nocturna, intermitente o continua) ha conducido a serias confusiones y hoy no se acepta.

Incontinencia urinaria: es una pérdida de orina involuntaria continua o intermitente, y esta última puede ser diurna o nocturna (enuresis).

Comentario: el término enuresis se utiliza para designar los escapes durante el sueño, independientemente de que existan o no síntomas urinarios diurnos. Los términos "enuresis diurna" para referirse a la incontinencia urinaria diurna o "síndrome enurético" para designar la incontinencia urinaria combinada día y noche deben evitarse. Si un paciente tuviera incontinencia urinaria combinada (diurna y nocturna intermitente), se etiquetará con dos diagnósticos: incontinencia urinaria diurna y enuresis.

Enuresis primaria: se considera que la enuresis es primaria si el niño nunca se ha mantenido seco durante un periodo mayor o igual a seis meses.

Enuresis secundaria: si la enuresis aparece después de un periodo seco de al menos seis meses. Enuresis monosintomática: si no existen síntomas diurnos que sugieran patología neurológica o urológica de base que altere la micción o continencia.

Comentario: la enuresis primaria monosintomática (EPM) puede coexistir con otras enfermedades que no alteren la función del aparato urinario (por ejemplo asma, diabetes mellitus...) y no por ello se considera no monosintomática.

Enuresis no monosintomática: es la que se acompaña de síntomas miccionales diurnos. Los síntomas relevantes para esta definición son: frecuencia miccional aumentada o disminuida (normal 2-8), incontinencia urinaria diurna, urgencia, retardo miccional, esfuerzo, chorro débil o intermitente, maniobras de retención urinaria, sensación de vaciado incompleto, goteo postmicional y dolor genital o del tracto urinario inferior. Estos síntomas sugieren patología neurológica-urológica, como síndrome de la vejiga hiperactiva (que engloba los de urgencia, frecuencia-urgencia intensa o frecuencia-urgenciaincontinencia), infecciones urinarias frecuentes, vejiga hipoactiva o micción obstructiva funcional. Comentario: la existencia de incontinencia urinaria diurna no es el único criterio para realizar el diagnóstico de no monosintomática.

Nocturia: es la manifestación de la necesidad de despertarse una o más veces para orinar. También puede referirse al número de micciones registradas durante un sueño nocturno (cada micción es precedida y seguida de un periodo de sueño).

Comentario: despertarse y orinar una vez cada noche entra dentro de lo normal. 
biales y vulvovaginitis en niñas, dermatitis, sospecha de abuso sexual). Manchas de la ropa interior (heces, secreciones u orina) pueden hacernos sospechar estreñimiento, infecciones o incontinencia diurna, diag- nósticos que en ocasiones los padres subestiman en la anamnesis.

- Espalda (lesiones que sugieran disrrafismo: nevus pilosos, fositas sacras por encima de la línea interglútea).

Tabla 2. Puntos clave del diagnóstico, clasificación y tratamiento de la enuresis

\begin{tabular}{l|c|c}
\hline Diagnóstico y clasificación & Sí & No \\
\hline Edad $\geq 5$ años & & \\
\hline Ha tenido algún periodo seco $\geq 6$ meses & Sí & No \\
\hline Antecedentes & & \\
\hline Apnea del sueño & & \\
\hline Estreñimiento crónico & & \\
\hline Infecciones urinarias repetidas & & \\
\hline Enfermedades del aparato urinario & & \\
\hline Enfermedades neurológicas & & \\
\hline Beber abundantes líquidos durante o tras la cena o noche* & Sí & No \\
\hline Ingesta de bebidas diuréticas (carbónicas, colas...) & & \\
\hline Síntomas urinarios diurnos & & \\
\hline Escapes de orina durante el día (incluso de gotas en la ropa interior) & & \\
\hline Posturas de retención habituales (contestar "no" si se debe a micción demorada**) & Sí & No \\
\hline Sensación de orinar con poca orina en la vejiga & & \\
\hline Segundo chorrito o gotas tras la micción & & \\
\hline Diario miccional & & \\
\hline VMMD $\leq 75 \%$ & & \\
\hline VMMD $\leq 45 \%$ & Sí & No \\
\hline Frecuencia miccional diurna $<2 * * *$ & & \\
\hline Frecuencia miccional diurna $>8 * * *$ & Sí & No \\
\hline Valoración de la gravedad de la enuresis & & \\
\hline Frecuencia de noches mojadas $<3$ a la semana & & \\
\hline Factores de fracaso del tratamiento con alarma & & \\
\hline Familia desestructurada o estrés familiar & No \\
\hline Niño poco motivado & & \\
\hline Familia poco motivada o poco colaboradora & & \\
\hline VMMD $\leq 45 \%$ & & \\
\hline Diagnóstico o sospecha de TDAH u otra alteración psiquiátrica & \\
\hline Factores de fracaso del tratamiento con desmopresina & \\
\hline VMMD $\leq 75 \%$ del esperado & & \\
\hline TDAH: trastorno por déficit de atención e hiperactividad; VMMD: volumen miccional máximo diurno. \\
*Riesgo de intoxicación hídrica en caso de tratamiento con desmopresina. \\
$* *$ Retención voluntaria e incontinencia por aplazamiento de la micción mientras está atrapado por el juego... \\
**Sospecha de alteración urológica. Aisladamente no tienen valor diagnóstico. \\
\hline
\end{tabular}


- Marcha talón-puntillas adelante y atrás descalzos (explora raíces sacras sensitivas y motoras que inervan la vejiga).

\section{El diario miccional}

La cumplimentación de un diario miccional durante 2-4 días (figura 1) se considera imprescindible ${ }^{3}$, ya que proporciona datos objetivos sobre aspectos valorados en la anamnesis, ayuda a detectar niños con enuresis no monosintomática, proporciona información sobre el pronóstico: volumen miccional máximo diurno (VMMD), frecuencia miccional elevada o disminuida, presencia de escapes urinarios, encopresis o estreñimiento, detecta niños que requieren más exploraciones y a familias con poca adherencia a las instrucciones.

\section{El registro de la defecación}

Algunos autores consideran también importante el registro de la defecación y sus características durante dos semanas, ya que se ha observado que en la entrevista los padres subestiman el estreñimiento ${ }^{6}$.

Ante EPM no se recomienda realizar ninguna prueba complementaria más. Si se sospecha enuresis no monosintomática, se debe remitir al hospital.

\section{Tratamiento de la enuresis primaria} monosintomática

Desmitificar el problema, evitar medidas punitivas, restringir líquidos vespertinos, evitando especialmente las bebidas diuréticas y colas, e ir al baño antes de acostarse son actitudes generales iniciales que muchas veces ya han realizado los padres.

Antes de instaurar el tratamiento se debe valorar con la familia y el niño el objetivo terapéutico y consensuar el más adecuado en cada caso. Si existen, se recomienda tratar el estreñimiento y la apnea del sueño. La terapia motivacional con calendarios (tratamiento conductual simple) ayuda a objetivar la situación basal de noches mojadas y se puede recomendar antes y junto a otros tratamientos.

En AP, además de las medidas generales ya descritas, se utiliza el tratamiento conductual con alarma y el farmacológico con desmopresina (figura 2). No se recomiendan otros fármacos como primera elección.

La mayoría de los niños con enuresis leve ( $<3$ noches/semana) tienden a la resolución espontánea. Sin embargo, la enuresis moderada (3-6/semana) o grave (diaria) y la que persiste a los diez años, difícilmente se resolverá sin tratamiento ${ }^{7}$. Independientemente de la gra- 
Figura 1. Diario miccional de tres días ${ }^{3}$.

\begin{tabular}{l} 
Nombre \\
Apellidos \\
\begin{tabular}{|l|l|l|l|l|l|l|l|l|}
\hline \\
\hline
\end{tabular} \\
\hline
\end{tabular}

\section{ANOTACIONES}

E: Escape, ha manchado (heces) o mojado (orina) la ropa

A: Aguanta hasta el límite porque está concentrado en un juego, película... y se olvida de ir al baño (orina o heces).

P: Prisa, deseo repentino, no le da tiempo a llegar al baño desde que nota el deseo de orinar o defecar, tiene que ir corriendo y se le puede escapar o se le escapa.
C: Va al baño y hace caca

$\mathrm{N}$ : Normales

D: Heces duras como de cabra

B: Blandas

\begin{tabular}{|l|l|}
\hline Volumen miccional máximo diurno (VMMD) de los tres días (excluyendo la 1. ${ }^{a}$ orina de la mañana) & $\mathrm{ml}$ \\
\hline VMMD teórico [(Edad +2$) \times 30]$ & $\mathrm{ml}$ \\
\hline$\%$ del VMMD teórico [VMMD $\times 100 / \mathrm{VMMD}$ teórico] & $\%$ \\
\hline ¿Ha habido escapes de orina diurnos? (Sí/No) & \\
\hline ¿Ha habido escapes de heces? (Sí/No) & \\
\hline ¿Hay estreñimiento? (Sí/No) & \\
\hline
\end{tabular}

Aclaraciones: el día comienza cuando se levanta el niño y termina cuando se levanta al día siguiente. Pueden apuntarse varios volúmenes o anotaciones en una misma casilla. Si se levanta a orinar por la noche, no se apunta el volumen, solo "Si" o "No". 
Figura 2. Algoritmo de tratamiento de la enuresis primaria monosintomática ${ }^{33}$

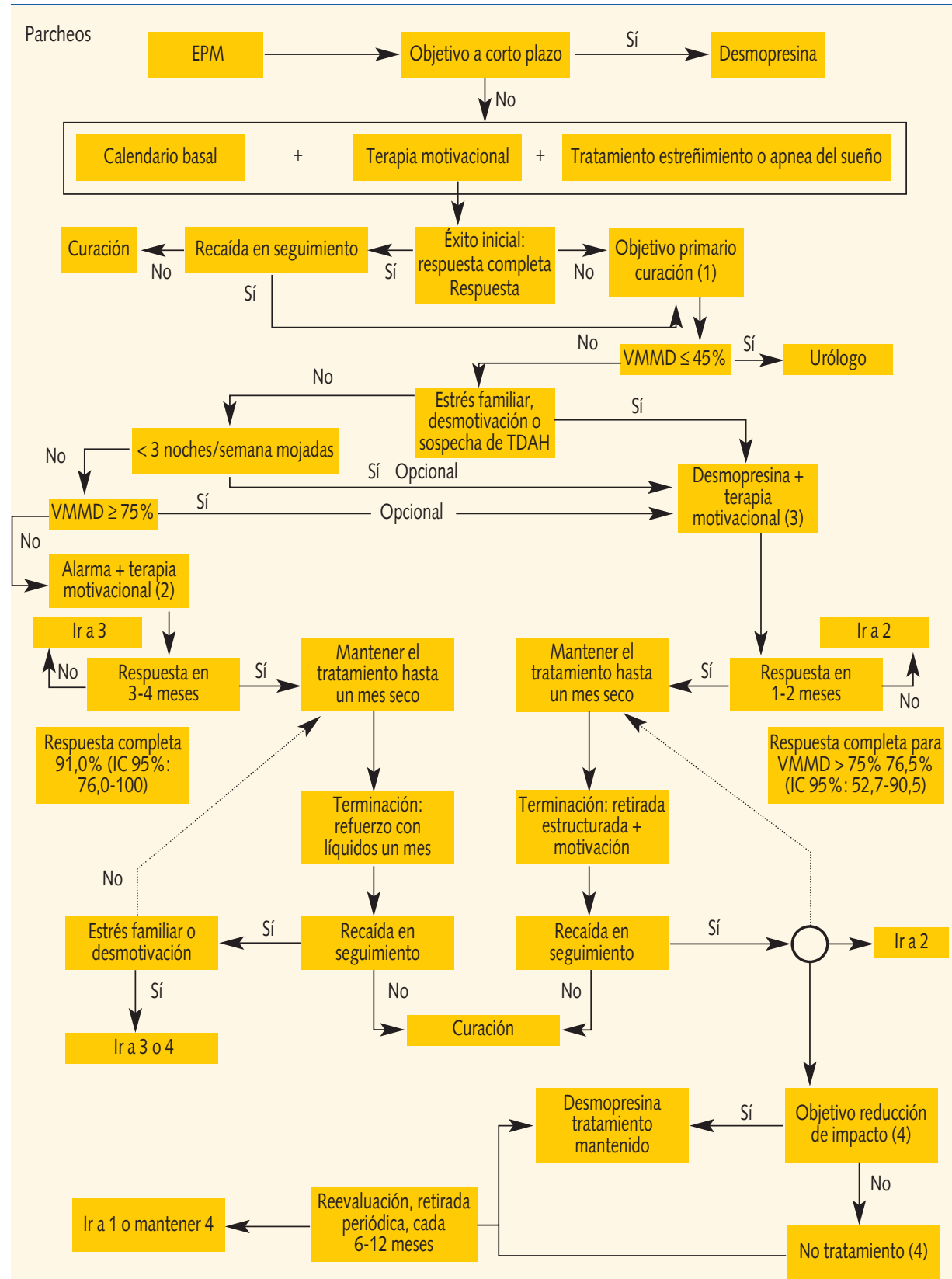

IC 95\%: intervalo de confianza del 95\%; TDAH: trastorno por déficit de atención e hiperactividad; VMMD: volumen miccional máximo diurno, se obtiene de los diarios miccionales. 
vedad y de la edad, el tratamiento también está indicado siempre que tenga repercusión negativa en el niño o la familia.

Usualmente el objetivo buscado es la curación, que significa sequedad completa definitiva tras finalizar el tratamiento. Sin embargo, cuando no es posible, puede ser adecuado el control de la enuresis con un tratamiento continuado a largo plazo, o a corto cuando interesa sequedad inmediata ante campamentos o situaciones esporádicas.

A la hora de valorar los resultados del tratamiento, hablaremos de "respuesta o fracaso" para referirnos a la situación de la enuresis mientras el paciente recibe tratamiento y de "curación o recaída" en referencia al resultado final, tras seis meses-dos años de finalizarlo.

\section{Tratamiento con alarma}

\section{¿Cuándo y cómo utilizar la alarma?}

Salvo excepciones, cuando buscamos curación, el tratamiento más eficaz y de elección es la alarma ${ }^{8}$. Sin embargo, la respuesta suele ser lenta y el cumplimiento exige esfuerzo e implicación del niño y la familia (tabla 3). Su eficacia es mayor cuando el número de noches mojadas es elevado, pero la clave del éxito depende fundamentalmente de la motivación e implicación tanto de los padres como del niño a lo largo del tratamiento y del estímulo que nosotros seamos capaces de ejercer sobre ellos con un seguimiento estrecho (aconsejable cada 2-3 semanas).

No se recomienda la alarma en casos de falta de motivación o colaboración de los padres o el niño, en situaciones estresantes del niño o la familia, ni ante el trastorno por déficit de atención e hiperactividad u otros problemas psiquiátricos, por ser factores de mal pronóstico para el éxito del tratamiento ${ }^{3}$. Tampoco está indicada cuando se busca una respuesta terapéutica rápida.

Si se utiliza la alarma, el riesgo relativo (RR) de conseguir 14 noches secas consecutivas es 13,3 veces mayor que sin ella (RR: 13,3; intervalo de confianza del 95\% [IC 95\%]: 5,6-31,5) y el de curación (no recaída), 5,5 veces mayor (RR: 5,56; IC 95\%: 1,54-20,0), el número necesario a tratar (NNT) es de 3, es decir que de cada tres niños a los que se les indique el tratamiento, uno curaráa $^{3,8}$.

\section{¿Cómo mejorar los resultados}

\section{de la alarma?}

Las cifras de curación con alarma mejoran considerablemente si se finaliza el tratamiento con una técnica de 
Tabla 3. Información sobre el tratamiento con alarma y desmopresina

Alarma

Son requisitos para el éxito de la alarma: la motivación, participación activa del paciente, su familia o cuidadores y el personal sanitario implicado.

Los padres y el niño deben conocer:

- Qué es una alarma para enuresis y cómo funciona.

- Que las tasas de curación son más elevadas que con cualquier otro tratamiento. La recaída es menor.

- Que el tratamiento suele ser largo (3-5 meses), salvo algunos casos de pocas semanas.

- Qué tienen que hacer: la alarma se dispara tras la emisión de las primeras gotas de orina; en ese momento, el niño debe despertarse y detener la micción, desconectar el sistema, ir al baño, cambiarse la ropa si se ha mojado y volver a conectarlo antes de acostarse. Ello exige la colaboración de la familia, sobre todo al principio, además de la implicación del niño. Motivar y felicitar el más mínimo progreso.

- Que puede despertar a otros miembros de la familia.

- Que no están financiadas por el Sistema Sanitario en la mayoría de la comunidades autónomas.

- Que no se debe desestimar el tratamiento con alarma ante la creencia de algunos padres de que su hijo será incapaz de despertarse con la alarma porque tiene un sueño muy profundo. La clave del éxito no es la intensidad del estímulo, sino la preparación del niño cada noche para despertarse y responder a la señal.

\section{Desmopresina}

- Usar preferentemente las formulaciones sublingual u oral. Reservar la nasal para cuando estas no sean posibles.

- Se recomienda usar la mínima dosis eficaz: 120-240 $\mu \mathrm{g}$ liofilizada sublingual o 0,2-0,4 mg oral.

- La formulación liofilizada no contiene lactosa.

- Evitar el uso concomitante con AINE y medicamentos que puedan inducir síndrome de secreción inadecuada de ADH: antidepresivos tricíclicos, inhibidores de la recaptación de serotonina, clorpromacina, carbamacepina, así como en niños con polidipsia o algunas patologías como la fibrosis quística que predisponen a la retención de líquidos o pérdida de electrolitos.

Los padres y el niño deben conocer:

- Que la respuesta es rápida y suele mantenerse mientras dura el tratamiento.

- Que las recaídas son frecuentes al suspender el tratamiento.

- Que se debe administrar la dosis una hora antes de realizar la última micción y acostarse.

- Que mayor dosis de la recomendada no produce mayor efecto sino mayor toxicidad. Especial vigilancia en niños con riesgo de olvidar que ya han tomado su medicación (repetición de dosis).

- Que no se deben administrar líquidos desde una hora antes de tomar la dosis hasta ocho horas después.

- Que deben suspender la administración de desmopresina si el niño presenta síntomas de retención hídrica (cefalea, náuseas, vómitos, ganancia de peso rápida, letargo o desorientación) e informar a su médico rápidamente.

- Que deben suspender temporalmente la administración de desmopresina si el niño presenta vómitos, diarrea o infecciones sistémicas que pueden aumentar el riesgo de hiponatremia.

- Que pueden permitir líquidos durante la comida del mediodía, pero deben restringirlos durante las dos horas previas a acostarse (adelantar la cena) y limitar los líquidos durante la cena a menos de $240 \mathrm{ml}$ (un vaso de agua) o más en aquellos niños que realizan deporte por la tarde o viven en zonas muy calurosas. No se debe administrar desmopresina los días que no ha sido posible restringir los fluidos.

AINE: antiinflamatorios no esteroideos; $A D H$ : horomona antidiurética. 
refuerzo. Consiste en prolongar el tratamiento administrando 1-2 vasos de agua antes de acostarse, tras haber logrado un mes de sequedad total, hasta conseguir nuevamente no mojar durante un $\mathrm{mes}^{8}$.

\section{Tipos de alarma}

Las alarmas de enuresis son mecanismos eléctricos que constan de un sensor de humedad que activa un emisor acústico, vibratorio o luminoso. No existe suficiente evidencia para decidir qué tipo de alarma es mejor, aunque no parece que existan diferencias entre ellas.

Existen alarmas de cama cuyo sensor tiene forma de esterilla que, colocada encima del colchón donde duerme e niño, hace disparar la alarma situada cerca de la cama. Tienen el inconveniente de ser poco transportables y de que detectan la humedad cuando el niño ha mojado completamente el pijama y la sábana.

Actualmente se recomiendan las alarmas portátiles, con cable o inalámbricas, ya que son de pequeño tamaño, más cómodas de utilizar y su sensor, colocado en la ropa interior o en el pijama del niño, capta precozmente la humedad de las primeras gotas de orina.

\section{Tratamiento farmacológico: desmopresina}

\section{¿Cuándo y cómo administrar desmopresina?}

Cuando no se puede utilizar la alarma, o si el objetivo es la sequedad a corto plazo, la desmopresina es el tratamiento de elección (figura 2). Es rápida y efectiva en reducir el número de noches mojadas mientras se toma, pero al suspenderla de forma brusca, la recaída es habitual'. En los niños que responden, la disminución del número de noches mojadas se observa en la primera semana y el efecto máximo a las cuatro.

El RR de no conseguir 14 noches secas consecutivas a la cuarta semana de tratamiento fue un $9 \%$ menor que con placebo (RR: 0,91; IC 95\%: 0,85-0,98) y el NNT fue de $12^{10,11}$. La efectividad que observamos cuando se administra durante corto tiempo, como en el caso de campamentos o pernoctaciones esporádicas fuera de casa, es mayor. Probablemente, la exigencia de un menor número de días de sequedad y la mayor motivación y preocupación del niño por el control de la enuresis favorezcan esta situación.

Al contrario que en la alarma, la frecuencia baja de noches mojadas a la semana predice una buena respuesta a 
desmopresina. Un volumen miccional máximo diurno $(\mathrm{VMMD}) \leq 75 \%$ del esperado es un factor desfavorable para este fármaco ${ }^{3}$.

La asociación de desmopresina a la alarma no ha mostrado beneficios a largo plazo y no se recomienda de forma rutinaria. Se puede usar junto con ella en niños que mojen más de una vez cada noche para reducir el número de micciones nocturnas y hacer más tolerable el tratamiento con alarma ${ }^{3}$.

\section{¿Cómo mejorar los resultados con desmopresina?}

Se ha descrito que una retirada estructurada disminuye las recidivas observadas con la interrupción brusca del tratamiento. Esta pauta se recomienda tras conseguir un mes de sequedad completa. Consiste en administrar la dosis completa de fármaco, disminuyendo quincenalmente los días de tratamiento hasta suspenderlo. Es una terapia conductual que añade un efecto curativo a la finalización del tratamiento con desmopresina ${ }^{12}$.

\section{Formas de presentación y dosis}

La desmopresina se presenta en formulaciones para administración intranasal, oral y sublingual. Debido al mayor riesgo de hiponatremia con la vía intranasal, se recomienda usar solo 1-2 comprimidos orales de 0,2 mg o liofilizados sublinguales de $120 \mu \mathrm{g}$ que son bioequivalentes ${ }^{13}$. Su efecto máximo se alcanza a la hora de la administración y se mantiene durante las horas de sue$\tilde{n} \mathrm{o}^{14}$.

La vía sublingual aventaja a la oral en que, por su mayor biodisponibilidad, requiere menos dosis, su absorción es más regular y se interfiere menos con los alimentos $^{14}$. No precisa agua para su toma $y$, al no exigir que el niño sepa tragar, es preferida por los más pequeños ${ }^{13}$.

\section{Precauciones con la desmopresina}

Si se tienen en cuenta unas precauciones básicas (tabla 3), es un fármaco seguro a corto y largo plazo. El efecto adverso más temido, que puede y debe evitarse, es la intoxicación acuosa ${ }^{15}$.

\section{Bibliografía}

1. Ramírez-Backhaus $M$, Martínez Agulló $E$, Arlándis Guzmán S, Gómez Pérez L, Delgado Oliva F, Martínez Garcia $\mathrm{R}$ et al. Prevalencia de la enuresis nocturna en la Comunidad Valenciana. Sección infantil del estudio nacional de incontinencia. Estudio EPICC. Actas Urol Esp. 2009; 33:1011-8.

2. Roquer JM, Capdevila J, Hortelano MA, Caveira R, Antoja P. Estudi de l'adquisicio del control vesi- 
cal nocturn i de la prevalença de l'enuresi nocturna en una ABS. Pediatr Catalana. 1999; 59:123-6.

3. Úbeda Sansano MI, Martínez García R, Díez Domingo J. Enuresis nocturna primaria monosintomática en Atención Primaria. Guía de práctica clínica basada en la evidencia. Rev Pediatr Aten Primaria. 2005;7(Suppl 3):s7-152.

4. Martínez García R, Mínguez Pérez M; Grupo Español de Urodinámica, SINUG. Propuestas de adaptación terminológica al español de la estandarización de la terminología del tracto urinario inferior en niños y adolescentes de la ICCS. Actas Urol Esp. 2008;32:371-89.

5. Neveus T, von Gontard A, Hoebeke P, Hjalmas K, Bauer S, Bower W et al. The standardization of terminology of lower urinary tract function in children and adolescents: report from the Standardisation Committee of the International Children's Continence Society. J Urol. 2006; 176:314-24.

6. McGrath $\mathrm{KH}$, Caldwell PH, Jones MP. The frequency of constipation in children with nocturnal enuresis: a comparison with parental reporting. J Paediatr Child Health. 2008;44(12):19-27.

7. Yeung CK, Sreedhar B, Sihoe JD, Sit FK, Lau $J$ J. Differences in characteristics of nocturnal enuresis between children and adolescents: a critical appraisal from a large epidemiological study. BJU Int. 2006;97:1069-73.

8. Glazener CM, Evans JH, Peto RE. Alarm interventions for nocturnal enuresis in children. Cochrane Database Syst Rev. 2005(2):CD002 911.
9. Glazener CM, Evans JH. Desmopressin for nocturnal enuresis in children. Cochrane Database Syst Rev. 2002(3):CD002112.

10. Skoog SJ, Stokes A, Turner KL. Oral desmopressin: a randomized double-blind placebo controlled study of effectiveness in children with primary nocturnal enuresis. J Urol. 1997;158(3 Pt 2):1035-40.

11. Schulman SL, Stokes A, Salzman PM. The efficacy and safety of oral desmopressin in children with primary nocturnal enuresis. J Urol. 2001;166:2427-31.

12. Butler RJ, Holland P, Robinson J. Examination of the structured withdrawal program to prevent relapse of nocturnal enuresis. J Urol. 2001;166:2463-6.

13. Lottmann H, Froeling F, Alloussi S, El-Radhi AS, Rittig S, Riis A et al. A randomised comparison of oral desmopressin lyophilisate (MELT) and tablet formulations in children and adolescents with primary nocturnal enuresis. Int J Clin Pract. 2007;61:1454-60.

14. Østerberg O, Savic RM, Karlsson MO, Simonsson US, Norgaard JP, Walle JV et al. Pharmacokinetics of desmopressin administrated as an oral lyophilisate dosage form in children with primary nocturnal enuresis and healthy adults. J Clin Pharmacol. 2006;46:1204-11.

15. Robson WL, Leung AK, Norgaard JP. The comparative safety of oral versus intranasal desmopressin for the treatment of children with nocturnal enuresis. J Urol. 2007;178(1): 24-30. 